\title{
$¿$ ¿Han sido seleccionados o se han acostumbrado? Ideas de estudiantes de biología sobre la selección natural y consistencia entre ellas
}

\author{
MARÍa PILAR JIMÉNEZ \\ Universidad de Santiago de Compostela \\ JOAQUIN FERNÁNDEZ \\ Universidad Complutense de Madrid

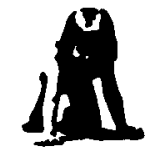 \\ Resumen
}

Estudiantes universitarios de Biología, ante problemas de respuesta abierta sobre la selección natural, utilizan, en porcentajes alarmantes, interpretaciones que consideran la adaptación como cambios beredables en los individuos. Se encontró un cierto grado de consistencia entre las respuestas de cada sujeto a los problemas en diferentes contextos; pero bay también casos en que una persona empleó esquemas conceptuales diferentes. Se sugiere que una de las condiciones para promover el cambio conceptual es que los profesores tengan en cuenta las ideas de los es. tudiantes.

Palabras clave: Esquemas conceptuales, Selección natural, Consistencia, Aprendizaje de la Biología.

\section{Have they been selected or have they become accustomed? Biology students' ideas on natural selection and the consistency between them.}

\section{Abstract}

When faced with open-response problems on natural selection there was a large percentage of university students of Biology who used explanations which regard adaptation as inberited changes in individuals that are passed on to offspring.

$A$ degree of consistency was found between subjects answers to problems with different task contexts; although sometimes more than one framework was used by the same subject.

It is suggested that one of the conditions for promoting conceptual change is that teachers pay attention to student's ideas.

Key words: Conceptual frameworks, Natural selection, Consistency, Biology learning.

Agradecimientos: Una primera versión de este trabajo fue presentada en el « 2 nd International Seminar on Misconceptions and Educational Strategies in Science and Mathematics» que tuvo lugar en Cornell en 1987, y al que pudo asistir la primera autora gracias a una bolsa del Comité Hispano-Norteamericano para la Cooperación Cultural y Educativa.

Agradecemos la colaboración de los profesores M. G." Barrutia y C. Benito.

Dirección del autor: Instituto de Ciencias de la Educación. Universidad de Santiago de Compostela y Universidad Complutense de Madrid.

Original recibido: Marzo 1988. Revisión recibida: Junio 1989. Aceptado: Junio 1989 


\section{INTRODUCCION}

Las investigaciones sobre esquemas conceptuales (también denominadas "preconcepciones» o «ideas alternativas» o "representaciones») iniciadas a finales de los años 70 con trabajos como los de Viennot (1977) y Driver (1981) han suministrado una ingente cantidad de datos sobre las dificultades experimentadas en el aprendizaje de los conceptos científicos, en concreto sobre las ideas -previas a la instrucción escolar- que los y las estudiantes de ciencias emplean para interpretar los fenómenos físicos y naturales, contradiciendo la concepción de los alumnos como "páginas en blanco».

Esta evidencia empírica viene a sumarse, como ha señalado Solomon (1987) a los enfoques de la epistemología constructivista y la psicología cognitiva que hacén hincapié en la construcción de modelos mentales, o «teorías personales» para explicar la realidad. En palabras de Claxton (1987)

«Todo lo que hacemos, vemos, sabemos o sentimos es fruto de dicha teoría. Incluso cuando ésta parece tan fina como una telaraña sigue existiendo y no hay manera de levantar el velo o mirar a hurtadillas alrededor de ella.»

Los resultados de una serie de estudios sobre estas ideas previas y sobre las dificultades para lograr el cambio hacia las aceptadas por la comunidad científica a través de la enseñanza, indican que estas ideas comparten algunos rasgos, que, según Driver et al. (1985) son:

* No coinciden con las aceptadas por la comunidad científica y enseñadas en la escuela («ciencia escolar»).

* Resultan difíciles de cambiar y, a pesar de la instrucción escolar, persisten en muchos casos en la edad adulta.

* Las mismas ideas son utilizadas por distintos individuos, en países diferentes y en un amplio abanico de edades.

En consecuencia el aprendizaje de la ciencia debe ser contemplado no como la adquisición de teorías nuevas, sino como el cambio de las existentes (Pozo 1987), es decir como un auténtico cambio conceptual (Hewson 1981, Carey 1985) que implicaría una reestructuración de las estructuras cognitivas (Hasweh 1986) y que, según Gil y Carrascosa, (1985) está vinculado a un cambio metodológico. Parte de las dificultades para que la reestructuración tenga lugar tienen relación con el hecho de que la evidencia experimental no es suficiente para provocar el abandono de las ideas alternativas. Otros factores implicados son (Hewson 1985) los compromisos epistemológicos de los estudiantes respecto a la generalización y la consistencia interna.

Aunque el número de trabajos sobre estas ideas previas en Biología es menor que en Física y Química, está creciendo rápidamente en los últimos años (Jiménez 1990, Serrano 1987). Wandersee et al. (1987) han encontrado 103 estudios en lengua inglesa, siendo uno de los contingentes más elevados el correspondiente a investigaciones sobre genética y evolución. 
Este estudio forma parte de una investigación más amplia sobre las ideas previas y los problemas en el aprendizaje de la selección natural.

\section{HIPOTESIS DE TRABAJO}

Las ideas de los y las estudiantes acerca de la evolución por el mecanismo de selección natural han sido investigadas entre otros por Brumby (1979, 1984), Deadman (1976) y Engel y Wood-Robinson (1985). En todos estos estudios aparece un amplio número de estudiantes que explican los cambios evolutivos de forma «lamarckista» por la heredabilidad de los caracteres adquiridos, e interpretan la adaptación no como un resultado de cambios en las poblaciones a lo largo de generaciones, sino como procesos graduales de transformación de los individuos.

El análisis de los escritos de Charles Darwin muestra que en una etapa inicial no descartaba la herencia de los caracteres adquiridos como mecanismo explicativo de los cambios observados en las especies (Gruber 1981), lo que puede ilustrar las dificultades que un cambio conceptual entraña.

Nuestro propósito, en este caso, es investigar las ideas a este respecto de los y las estudiantes universitarios de Biología, en particular los siguientes aspectos:

1. 9 Persistencia de los esquemas alternativos en este grupo, que, por su opción profesional, consideramos seleccionado.

2.9 Consistencia en la utilización de un mismo esquema en diferentes problemas por cada individuo.

3. 9 Diferencias - o no- relacionadas con el género en el uso de los esquemas alternativos y científicamente aceptados.

Nuestras hipótesis iniciales para cada una de las cuestiones eran:

1. Existencia de un porcentaje elevado de estudiantes universitarios de Biología que mantienen los esquemas alternativos.

2.. Un cierto grado de consistencia en las respuestas, pero no en todos los individuos.

3. ${ }^{2}$ Un menor empleo de las ideas científicamente aceptadas por parte de las alumnas.

\section{METODOLOGIA}

La muestra comprende dos grupos - de un total de seis- de 76 y 81 estudiantes respectivamente, que cursaban 2.9 de Ciencias Biológicas en la Universidad Complutense de Madrid en 1986-87. Como la asignación de estudiantes a los grupos es alfabética, creemos que son representativos del total. El mecanismo de la selección natural se estudia en COU y también forma parte del programa de primer curso en la Facultad. 
Tras pedirles su colaboración - voluntaria- en un trabajo de investigación sobre problemas en el aprendizaje de la Biología, se les entregó un juego de tres hojas, cada una con un problema relacionado con el tema en cuestión. Las palabras «evolución», «selección natural» y «adaptación» no aparecen en los enunciados, ni fueron mencionadas en la advertencia, ya que tratábamos de evitar meras repeticiones de definiciones sacadas de libros, que muestran conocimientos del tipo que Lawson (1988) llama declarativo. Las hojas fueron grapadas en distinto orden de forma que unas veces apareciera un problema en primer lugar y otras otro. Se trataba de una prueba de respuesta abierta y sin limitación de tiempo (de hecho todos contestaron en menos de una hora).

Los tres problemas figuran en el apéndice 1. Comentaremos brevemente algunas de sus características:

- Se trata de problemas de aplicación en que se pretende averiguar el conocimiento procedimental de los y las estudiantes, pidiéndoles interpretaciones o predicciones sobre fenómenos cotidianos o que por su contexto resultan familiares.

- Los problemas sobre «Piojos» y «Bacterias» son muy similares en su construcción y esperábamos que los estudiantes los respondiesen de forma parecida. El de los «Piojos» cita una información del diario «El País», solicitando una explicación de la misma, y el de las «Bacterias» está tomado de las Pruebas de Acceso a la Universidad de Santiago en 1986.

- El problema sobre los «Ratones», basado en las experiencias de Weissman, fue elaborado para ser utilizado en clase, como parte de unas actividades que pretenden lograr un aprendizaje significativo. Nuestra hipótesis sobre este problema era que al ser el organismo implicado un vertebrado, y la característica adquirida - cortarle la colauna mutilación que, de acuerdo con la experiencia, los estudiantes saben que no se transmite, las respuestas serían similares a las aceptadas por la ciencia escolar en un porcentaje mayor que en los otros dos problemas.

\section{ANALISIS DE DATOS: PERSISTENCIA DE IDEAS ALTERNATIVAS}

Con posterioridad al análisis hemos clasificado las respuestas en tres categorías, excluyentes entre sí:

A. Interpretaciones acordes con la ciencia escolar, o que contienen elementos de la misma (es decir que explican la resistencia de piojos y bacterias por supervivencia diferencial, y que predicen que los ratones seguirán naciendo con cola).

B. Respuestas basadas en adaptaciones post hoc y transmisión de las características adquiridas a la descendencia (resistencia o falta de cola).

C. Otro tipo de explicaciones, incluyendo las teológicas, intencionales, no codificables y «no contestan». 
TABLA I

Categorias y subcategorias de respuestas

A. Respuestas coincidentes con la ciencia escolar

A 1. Incluyendo variabilidad y supervivencia diferencial

A 2. Incluyendo sólo parte de la explicación científicamente aceptada

B. Basadas en reacciones a factores ambientales y/o herencia de los caracteres adquiridos.

B 1. Cambios genéticos en respuesta a y dirigidos hacia un factor ambiental determinado

B 2. El organismo individual «se acostumbra», «se habitúa»...

B 3. El organismo «se hace inmune» y transmite este carácter.

C. Otras

C 1. Explicaciones teleológicas «para la supervivencia de la especie»

C 2. Explicaciones intencionales/antropomórficas.

C 3. No codificables.

C 4. No contesta.

Además se establecieron subcategorías, que se muestran en la Tabla I. Respecto a las categorías y subcategorías debemos señalar que:

- El motivo de incluir respuestas un tanto heterogéneas en la categoría $\mathrm{C}$, responde a nuestra intención de comparar las explicaciones acordes con la ciencia escolar $\mathrm{A}$, con la idea alternativa más frecuente $\mathrm{B}$. - Por esta misma razón establecimos una jerarquización, de forma que las respuestas individuales que incluían elementos de $\mathrm{B}$ y de $\mathrm{C}$ fueron categorizadas como $\mathrm{B}$, aunque en la Tabla 3 hemos incluido los datos correspondientes a estas respuestas «dobles».

- Hemos incluido en la categoría B las explicaciones basadas en la adquisición de inmunidad, porque -independientemente de revelar una concepción errónea de lo que es la inmunidad, tan extendida que alcanza incluso a investigadores como Brumby (1984) que emplean expresiones como «insectos que se inmunizan»- nos parecen relacionadas con la idea de reacción a posteriori del ofganismo frente a los factores ambientales y la transmisión de los rasgos así adquiridos a la descendencia. Somos conscientes de que esta inclusión difiere de la categorización de otros estudios (Brumby 1979).

- En cualquier caso, siendo el contexto del problema sobre «Ratones» muy distinto de los otros dos esta subcategoría de «inmunidad» B 3 no le es aplicable.

- Llamamos explicaciones «intencionales» («psicológicas» de acuerdo con la denominación de Susan Carey 1985) a aquéllas en que los procesos de cambio son interpretados como aspectos del comportamiento humano de carácter intencional, es decir en términos de los deseos y creencias del sujeto. Carey opone estas interpretaciones a las «biológicas». Otros autores las denominan «antropomórficas», pero consideramos relevante la distinción, por cuanto subraya el hecho de que tampoco la especie humana puede inmunizarse o modificar un rasgo anatómico de forma que sea hereditario por un acto de voluntad. 
- Hemos incluido las respuestas que explicaban el aumento de bacterias resistentes únicamente por conjugación bacteriana en la categoría C 3 por entender - de acuerdo con los especialistas en Genética consultados- que este mecanismo podría explicar tan sólo un pequeño incremento, siendo la supervivencia diferencial responsable del más notable.

- En el caso de respuestas cuya interpretación resultase ambigua o dudosa entre $\mathrm{A}$ y $\mathrm{B}$, o entre $\mathrm{A}$ y $\mathrm{C}$, hemos considerado siempre la hipótesis más desfavorable para nuestra hipótesis (y más favorable para el sujeto), categorizándolas como $A$.

Algunos ejemplos de respuestas y las categorías asignadas figuran en el Apéndice 2.

El proceso de categorización de las respuestas fue realizado de forma independiente por las dos personas firmantes, alcanzándose un nivel de acuerdo del $90 \%$ en las tres grandes categorías.

TABLA II

Número y porcentaje de respuestas (grandes categorías) $N=157$

\begin{tabular}{cccc}
\hline Problema/Categoría & $\mathbf{N}^{0}$ Estudiantes & \% de Estudiantes & $\sigma$ \\
\hline BACTERIAS & & & \\
A & 78 & 49.68 & 3.99 \\
B & 58 & 36.94 & \\
C & 21 & 13.37 & \\
\hline & & & 3.69 \\
PIOJOS & & & \\
A & 50 & 31.18 & \\
B & 105 & 66.87 & \\
C & 2 & 1.27 & \\
\hline & & & \\
RATONES & & & \\
A & 93 & 59.23 & \\
B & 56 & 35.66 & \\
C & 8 & 5.09 & \\
\hline
\end{tabular}

En la Tabla II aparece el número, porcentaje y $\sigma$ (desviación estándar) de las respuestas asignadas a las tres categorías para cada problema, y en la Tabla III el número de respuestas para cada subcategoría. Respecto al total de los y las estudiantes (157) señalaremos que:

a) Como se observa en la Tabla 2 el porcentaje de estudiantes que utilizan el esquema acorde con la ciencia escolar varía de $59,23 \%$ en el problema de «Ratones» a 31,18\% en el de «Piojos». De acuerdo con nuestra hipótesis esperábamos un mayor uso del esquema aceptado por la ciencia en el problema de «Ratones», al ser el organismo un vertebrado y la situación asimilable a la de un ser humano que sufre una mutilación. Algunos estudiantes - por ejemplo el núm. 57* en el 
TABLA III

Número de respuestas asignadas a las subcategorias (entre paréntesis las codificadas en otra)

\begin{tabular}{llll}
\hline Subcategoría & Bacterias & Piojos & Ratones \\
\hline A 1. Supervivencia diferencial & 53 & 34 & 58 \\
A 2. Parte del esquema aceptado & 25 & 16 & 35 \\
& & & \\
B 1. El entorno causa cambio genético & $22(+1)$ & $33(+1)$ & 21 \\
B 2. Se acostumbran & $27(+4)$ & $41(+2)$ & 35 \\
B 3. Se vuelven inmunes & $9(+1)$ & $31(+9)$ & - \\
C 1. Teleológicas & $7(+10)$ & $1(+11)$ & $6(+9)$ \\
C 2. Intencionales/antropomórficas & $1(+2)$ & $-(+5)$ & $2(+1)$ \\
C 3. No codificables & 9 & 1 & - \\
C 4. No contesta & 4 & - & - \\
\hline
\end{tabular}

Apéndice 2- mencionan la pérdida de la cola como un cambio «morfológico» que «no afecta a los genes». Esta oposición entre morfología y fisiología en relación con las transformaciones genéticas requeriría ser investigada con detalle.

Este porcentaje relativamente más elevado de respuestas acordes con la ciencia escolar se ve acentuado si tenemos en cuenta que, como el problema de «Ratones» tiene dos partes, las respuestas fueron categorizadas como A sólo cuando este esquema se utilizaba en la respuesta a ambas.

b) Lo que no parecía esperable, de acuerdo con nuestra hipótesis, era la diferencia entre las respuestas de acuerdo con la ciencia escolar a «Bacterias» $(49,68 \%)$ y a «Piojos» $(31,18 \%)$. Este último problema resultó ser el más difícil, y el número de estudiantes que utilizan esquemas de la categoría B - relacionados con la transmisión a la descendencia de caracteres adquiridos-es muy elevado (66,87\%). El valor de $\chi^{2}$ que resulta de comparar las respuestas a «Bacterias» y «Piojos» es de $10,34 \%$, significativo mucho más allá del nivel de 0,01 .

Estos resultados avalan la opinión de Engel y Driver (1986) de que problemas similares a ojos de los científicos - $-\mathrm{y}$, añadimos nosotros, de profesoras y profesores - pueden no parecer semejantes a los estudiantes.

Quizá esta diferencia en el uso de un esquema u otro en la resolución de los problemas de «Bacterias» $\mathrm{y}$ «Piojos» esté relacionada con el contexto "científico» (un cultivo en laboratorio) del primero, por oposición al contexto cotidiano del segundo. Como señala Solomon (1987) los contextos que recuerdan situaciones familiares, en las que sería de esperar la aplicación del conocimiento cotidiano, dificultan la utilización del razonamiento lógico y el conocimiento científico. Esta misma autora indica que, a pesar de estos problemas, la enseñanza de una ciencia aislada del contexto cotidiano no es una opción válida. La necesidad de utilizar en clase ejemplos y problemas cercanos a la vida 
diaria subsiste, si bien debemos ser conscientes de las dificultades que conlleva.

c) En nuestra opinión el hecho de que sólo el 59,23\% de los y las estudiantes -en el problema «más fácil»- y el 31,18\% —en el caso de los «Piojos»- utilicen el esquema de la ciencia escolar es un motivo de seria preocupación. Más aún si tenemos en cuenta que hay un cierto número de estudiantes que dan interpretaciones teleológicas, e incluso algunos que ofrecen explicaciones de carácter intencional. Recordemos que al ser las respuestas asignadas a categorías excluyentes, las que incluían por ejemplo B 1 y C 2 se codificaron sólo como B 1 . En la Tabla III, junto a $\mathrm{C} 1$ y $\mathrm{C} 2$ aparece entre paréntesis el número de respuestas de carácter teleológico e intencional dadas en combinación con otras categorías, y la misma notación se emplea en B. Estos números entre paréntesis no se consideran al calcular los porcentajes. Teniendo en cuenta que se trata de un grupo seleccionado, que pretende obtener una licenciatura en Biología $-y$ posiblemente enseñarla a otras personas- y reconociendo la importancia de la selección natural y los mecanismos hereditarios en la Biología moderna nos parece que estos datos son indicio de una situación grave que requiere estudios más detallados y un tipo de estrategias docentes que tengan en cuenta estos problemas.

\section{CONSISTENCIA EN ELUSO DE ESQUEMAS CONCEPTUALES EN LAS RESPUESTAS INDIVIDUALES}

En su tesis doctoral, Brumby (1979) señalaba algunas inconsistencias entre respuestas individuales. Un trabajo modélico sobre este tema ha sido publicado por Engel y Driver (1986). Estas autoras encuentran un cierto grado de consistencia entre las respuestas, sugiriendo, como ya hemos comentado, que el contexto y la redacción de los problemas pueden ser responsables de parte de las inconsistencias, ya que lo que a ojos de la persona que investiga son distintas formas de un mismo problema, pueden aparecer para los estudiantes como problemas distintos.

Para estudiar la consistencia en los esquemas usados por cada persona, realizamos tablas de contingencia para cada par de problemas. En la Tabla 4 aparece, a título de ejemplo, la tabla de contingencia para «Piojos» $y$ «Bacterias». Por razones de espacio, para los otros dos pares de problemas damos sólo el coeficiente de contingencia $\mathrm{C}$, y el $\chi^{2}$ que indica el nivel de significación estadística que, para 4 grados de libertad, es en los tres casos, significativo al nivel de 0,01 (Tabla 5).

Como se observa en las Tablas 4 y 5 existe una correlación positiva entre las respuestas que utilizan los esquemas alternativo y aceptado en cada par de problemas, siendo la más elevada entre «Bacterias» y «Piojos», tal y como esperábamos. De hecho todos los estudiantes que utilizaron el esquema aceptado para «Piojos» hicieron lo propio para «Bacterias» (no a la inversa).

Para comparar la consistencia en el uso de los esquemas aceptado 
TABLA IV

Tabla de contingencia $(3 \times 3)$ para "Bacterias" $y$ "Piojos».

Entre paréntesis los valores de independencia. Coefic. $C=0.54$

\begin{tabular}{lcccr}
\hline $\begin{array}{l}\text { Bacterias/ } \\
\text { Piojos }\end{array}$ & $\begin{array}{c}\text { A Esquema } \\
\text { aceptado }\end{array}$ & $\begin{array}{c}\text { B Adaptación } \\
\text { post hoc }\end{array}$ & C Otras & $\begin{array}{r}\text { Total } \\
\text { Piojos }\end{array}$ \\
\hline A esq. acept. & $48(24.84)$ & $1(18.47)$ & $1(6.68)$ & 50 \\
\hline B adapt. post. & $30(52.16)$ & $56(38.78)$ & $19(14.04)$ & 105 \\
\hline C otras & $0(0.99)$ & $1(0.73)$ & $1(0.26)$ & 2 \\
\hline Total Bact. & 78 & 58 & 21 & $\mathrm{~N}=157$ \\
\hline
\end{tabular}

Tabla V

Coeficiente de contingencia y $\chi^{2}$ para cada par de problemas. $N=157$

\begin{tabular}{lcc}
\hline Problemas & \multicolumn{2}{c}{ Coeficiente de contingencia C } \\
\hline Bacterias y Piojos & 0.54 & 64.62 \\
Bacterias y Ratones & 0.43 & 35.61 \\
Piojos y Ratones & 0.32 & 17.91 \\
\hline
\end{tabular}

y alternativo, calculamos los coeficientes de contingencia $C$ para las subcategorías de $\mathrm{A}$ y $\mathrm{B}$, realizando tablas de contingencia $(2 \times 2)$ para A 1 y A 2 y para B 1 y B 2 . Como ya hemos mencionado, B 3 es aplicable sólo al par «Bacterias» y «Piojos», como se ve en la Tabla VI, en la que $\mathbf{N}=56$, que son los y las estudiantes que utilizaron el esquema categorizado como $B$ en ambos problemas. Los valores del coeficiente de contingencia para los restantes problemas y categorías figuran en la Tabla VII.

Debe tenerse en cuenta que, mientras que las subcategorías $B$, B 2 y B 3 pueden considerarse excluyentes, A 2 fue asignada a respuestas que contenían elementos de la explicación acorde con la ciencia escolar, de forma que la correlación entre A 1 y A 2 tal vez no tiene el mismo significado que la correlación entre el empleo de las subcategorías de B. Teniendo en cuenta esto y el tamaño de la muestra, estos resultados deben considerarse como una primera aproximación al problema.

Como se ve en la Tabla VII no se pueden extraer conclusiones respecto al uso de los esquemas alternativo o aceptado. Lo que sí parece claro es que cuando los y las estudiantes perciben dos problemas como similares (como es el caso con «Bacterias» y «Piojos») aparece un alto grado de consistencia en el empleo de los esquemas.

En todos los casos aparecen correlaciones positivas, lo que consti- 
TABLA VI

Tabla de contingencia $(3 \times 3)$ para las subcategorias B en los problemas "Bacterias" $y$ «Piojos». $N=56 . C=0.72$

\begin{tabular}{lcccc}
\hline $\begin{array}{l}\text { Bacterias/ } \\
\text { Piojos }\end{array}$ & B 1 & B 2 & B 3 & Total Piojos \\
\hline B 1 & $13(6)$ & $3(7.42)$ & $0(2.57)$ & 16 \\
\hline B 2 & $6(9.37)$ & $17(11.60)$ & $2(4.01)$ & 25 \\
\hline B 3 & $2(5.62)$ & $6(6.96)$ & $7(2.4)$ & 15 \\
\hline Total Bact. & 21 & 26 & 9 & $\mathrm{~N}=56$ \\
\hline
\end{tabular}

TABLA VII

Coeficiente de contingencia $C$ para las subcategorias de $A$ y $B$

\begin{tabular}{|c|c|c|c|c|c|}
\hline \multirow[t]{2}{*}{ Problemas } & \multicolumn{2}{|c|}{ A 1 y A 2} & \multicolumn{3}{|c|}{ B 1 y B $2(*$ y B 3$)$} \\
\hline & $\mathbf{N}$ & C & $\mathbf{N}$ & & C \\
\hline Bacterias y Piojos & 48 & 0.31 & 56 & * & 0.72 \\
\hline Bacterias y Ratones & 63 & 0.12 & 32 & & 0.25 \\
\hline Piojos y Ratones & 42 & 0.41 & 35 & & 0.15 \\
\hline
\end{tabular}

tuye un dato más a favor de la existencia real de los esquemas conceptuales, que estarían lejos de ser construcciones «ad hoc» o artificios provocados por la investigación, como ha sido sugerido por algunos autores (Mc Clelland 1984).

\section{DIFERENCIAS ENTRE CHICAS Y CHICOS}

Las diferencias entre ambos sexos en los resultados académicos en las asignaturas de ciencias y en las opciones profesionales han sido tratadas por diversos autores, por ejemplo Erickson y Erickson (1984) y, recientemente el International Journal of Science Education (1987) ha dedicado un número especial a este tema. Estas diferencias están relacionadas con una disminución en las actitudes positivas hacia la ciencia a lo largo de la escolarización, por lo que no es extraño que un número relativamente pequeño de mujeres escoja carreras de ciencia e ingeniería en la universidad. Se han realizado algunas propuestas y proyectos en distintos países para modificar esta situación (Kahle 1985, Kelly 1987).

El estudio de las diferencias entre géneros en nuestro caso fue posible solamente con uno de los grupos $(N=76)$, ya que en el otro las hojas (que se respondian de forma anónima) fueron recogidas de un modo que no nos permitó agruparlas por separado. 
TABLA VIII

Diferencias entre chicas $(N=41)$ y chicos $(N=35) . N=76$

\begin{tabular}{|c|c|c|c|c|c|}
\hline \multirow{2}{*}{$\begin{array}{l}\text { Género } \\
\text { Categorías/ } \\
\text { Problemas }\end{array}$} & \multicolumn{2}{|c|}{ CHICAS } & \multicolumn{2}{|c|}{ CHICOS } & \\
\hline & Acept. & Otras & Acept. & Otrás & \\
\hline Bacterias & 15 & 26 & 25 & 10 & 9.19 \\
\hline Piojos & 9 & 32 & 18 & 17 & 7.16 \\
\hline Ratones & 10 & 31 & 26 & 9 & 18.85 \\
\hline
\end{tabular}

Para comprobar si existían diferencias entre el número de chicas y chicos en el uso de los esquemas, calculamos el $\chi^{2}$ para cada problema. Los valores de la Tabla VIII muestran que en todos ellos los chicos utilizaron el esquema acorde con la ciencia escolar en una proporción más alta que las chicas. Más aún, en los tres primeros el número de chicos que utilizaron el esquema aceptado fue más alto que el de los que no lo usaron, mientras que para las chicas la situación es la contraria. En todos los casos los valores del $\chi^{2}$ son significativos al nivel de 0,01 .

Sin embargo, un análisis similar (Jiménez y Fernández 1987) con estudiantes de COU no mostró diferencias significativas. Quizá la discordancia entre unos resultados y otros pueda deberse a que los estudiantes universitarios, por la asignación alfabética a los grupos, pueden considerarse como una muestra al azar, mientras que los de C.O.U. eran, en todos los casos, alumnos de profesores y profesores implicados en actividades de renovación y/o investigación. Kahle (1985) ha mostrado la equiparación en rendimiento e interés en las ciencias -e incluso en aspectos como la independencia de campo, la aptitud espacial o el locus de control- entre estudiantes mujeres y varones que tenían este profesorado que los anglosajones denominan «excelente». En todo caso, se trata de un tema apenas investigado en nuestro país, y en el que será necesario profundizar en el futuro.

\section{RESUMEN Y CONCLUSIONES}

Enfrentados a problemas sobre la selección natural, un porcentaje apreciable de estudiantes universitarios de Biología utilizaron explicaciones en las que se contempla la adaptación como cambios en los individuos que son transmitidos a la descendencia, y algunos de ellos creen que «los miembros que no se utilizan acabarán por desaparecer» o que «se atrofiarán». Algunos incluso explican los cambios evolutivos con expresiones teleológicas como "para sobrevivir» o intencionales.

Algunos estudiantes emplean un solo esquema, pero otros mezclan varios, mostrando un grado de confusión considerable. Quizá una consecuencia que se derive de estos datos sea que la complejidad indudable del tema y la necesidad de tiempo suficiente para llevar a cabo actividades de aprendizaje diseñadas para lograr el cambio conceptual 
demandarían una reducción en los programas de enseñanza secundaria, ya que programas largos favorecen el empleo de estrategias de memorización mecánica. Aquí hemos explorado únicamente el tema de la selección natural, pero seguramente la situación será parecida en otros, como muestran Cañal y Rasilla (1986) en el caso de la fotosíntesis.

Los porcentajes de estudiantes que utilizaron los esquemas aceptado y alternativo difieren en los distintos problemas, pero existe un cierto grado de consistencia entre las respuestas de cada persona en distintos contextos, $y$, aunque las ideas alternativas varían, la mayor parte de los y las estudiantes utilizaron un número relativamente pequeño de las mismas. Esto puede facilitar la preparación de materiales y actividades de enseñanza que cuenten con estos esquemas alternativos que son usados por un gran número de estudiantes. Nos parece que una de las condiciones para promover el cambio conceptual es la de que los profesores y profesoras tengan en cuenta estas ideas, hipótesis que requiere ser comprobada por la investigación.

El hecho de que un número significativo de personas utilizase unas veces el esquema aceptado, y otras el alternativo para interpretar cada problema plantea cuestiones como la importancia del contexto (científico o cotidiano). Otra posible explicación de las inconsistencias podrían ser los intentos del o la estudiante por compatibilizar el esquema conceptual alternativo con datos de su experiencia, o fragmentos de conocimientos científicos, como el $57^{*}$ en el apéndice 2. Lo que parece claro es que la coherencia entre distintos aspectos de una teoría científica es percibida como necesaria por la comunidad científica, pero no por los estudiantes, problema cuya relevancia ha sido señalada por Hewson (1985), y quizá esto significa que estos alumnos no entienden que están utilizando dos teorías distintas («lamarckismo» y «darwinismo») y que ambas no pueden servir para explicar la realidad simultáneamente. En la actualidad estamos tratando de investigar con más detalle el papel jugado en el aprendizaje por esta comparación explícita de dos modelos.

Los varones de esta muestra obtuvieron resultados significativamente mejores que las mujeres. Esto, que confirma trabajos de otros autores en diversos países, indica que se deben emprender acciones con el objetivo de aumentar el interés y la participación de las chicas en la ciencia.

\section{Referencias}

Brumby, M. (1979). «Student's Perceptions and learning styles associated with the concept of evolution by natural selection». Tesis Doctoral, Univ. de Surrey.

BRUMBY, M. (1984). «Misconceptions about the concept of natural Selection by medical biology students», Scie. Educ., 68 (4): 493-503.

Cañal, P., y Rasilla, C. (1986). «Une étude sur le niveau de structuration des concepts "photosynthese" et "respiration" des étudiants de l'Ecole Normale». En A. Giordan \& J.L. Martinand (Eds.), Feuilles d'Epistémologie appliquée et de Didactique des Sciences 8: 39-44.

CAREY, S. (1985). Conceptual Change in Childbood, A Bradfordbood. The MIT Press.

Claxton, G. (1987). Vivir y Aprender, Alianza Psicología. Traducción de Live and Learn Harper \& Row. London: 1984. 
DeAdman, J.A. (1976). «The structure and development of concepts associated with the topic of evolution in secondary school boys». Tesis Doctoral. Univ. de Londres. Chelsea College.

DrIver, R. (1981). «Pupils' Alternative frameworks in Science». Eur. J. Scie. Ed. 3: 93-101.

DRIVER, R.; GUESNE, E \& THIBERGIEN, A. (1985). Children's ideas in Science. Open University Press. London. Trad. al castellano como Ideas cientificas en la infancia y la adolescencia. Coed. MEC, Morata. 1989.

ENGEL, E.C. \& DRIVER, R. (1986). «A Study of Consistency in the Use of Student's Conceptual Frameworks Across Different Task Context». Scie. Educ. 70 (4): 473-496.

ENGEL, E.C. \& WOOD-ROBINSON, C. (1985). «How secondary students interpret instances of biological adaptation». J. Biol. Educ., 19 (2): $125 \cdot 130$

ERICKSON, G. \& ERICKSON, L. (1984). «Females and Science Achievement: Evidence, explanations and implications». Scie. Educ., 68 (2): 63-89.

GIL, D. \& CARRASCOSA J. (1985). «Science learning as a conceptual and methodological change». Eur. J. Scie. Educ., 7 (3): 231-236.

GrUBER, H. (1981). Darwin on Man: A psychological study of scientific creativity. A Phoenix Book.. University of Chicago Press. Trad. Darwin sobre el bombre, Alianza 1984.

HASWEH, M. (1985). «Toward an explanation of conceptual change». Eur. J. Scie. Educ., 8 (3): 229-249.

HEwSON, P. (1981). «A conceptual change approach to learning science». Eur. J. Scie. Educ., 3 (4): 383-396.

HEwSON, P. (1985). «Epistemological commitments in the learning of Science: Examples from dynamics». Eur. J. Scie. Educ., 7 (2): 163-172.

Internattonal Journal Of SCIENCE Education. (1987). «Special Issue: Gender and Science». $9(3)$.

JiMÉNEZ ALEIXANDRE, M.P. (1990). Los seres vivos vistos por los adolescentes, Coed. Laia, MEC

JiménEZ, M.P. \& FERNÁNDEZ, J. (1987). «'Pueden las bacterias mutar cuando les conviene?» Algunas ideas de los alumnos/as de COU sobre la Selección Natural. Actas II Congreso Int. Investig. Didáctica ciencias y Matemáticas, Valencia, pp. 115-116.

KaHLE, J.B. (1985). Women in Science. A Report from the Field. Palmer Press. London.

Kelly, A. (Ed) (1986). Science for girls?. Open University Press. London.

LAwSON, A. (1988). «The acquisition of biological knowledge during childhood: conceptual conflict or tabula rasa?». J. Res. in Scie. Teach., 25 (3): 185-199.

MCCLELLAND, J.A. (1984). «Alternative Frameworks: Interpretation of Evidence». Eur. J. Scie. Educ., 6 (1), 1-6.

Pozo, J.I. (1987). Aprendizaje de la Ciencia y Pensamiento Causal. Visor. Madrid.

SERRANO, M.T. (1987). «Representaciones de los alumnos de Biología: estado de la cuestión y problemas para su investigación en el aula». Enseñanza de las Ciencias, 5 (3): 181-188.

SOLOMON, J. (1987). «Social influences on the construction of Pupils' understanding of Science». Studies in Science Education, 14: 63-82.

VIENNOT, L. (1977). «Le raisonnement spontané en Dynamique élémentaire». Tesis doctoral Universidad de París VIl.

Wanderseee, J; Mintzes, J. \& ARnAudin, M.J. (1987). «Childrens’ Biology: a Content Analysis of Conceptual Development in the Life Sciences. 2nd Int. Sem. on Misconceptions \& Educational Strategies in Science \& Mathematics. Cornell.

\section{Apéndices}

\section{APENDICE 1. LOS PROBLEMAS}

\section{1. «BACTERIAS»}

Una mutación poco frecuente en la bacteria Eschericbia coli causa resistencia a la estreptomicina. Si se cultiva Escherichia coli en un medio con estreptomicina se observa un gran aumento en el número de bacterias resistentes.

¿Cambia la estreptomicina la tasa de mutación?

¿Cómo explicas estos hechos?

\section{2. «PIOJOS"}

\section{Del diario El Pais.}

«Un $15 \%$ ó un $20 \%$ de escolares sufre ataques de piojos entre otoño y Semana Santa. No se conocen con exactitud las causas de las recientes epidemias, ya que la higiene ha mejorado, pero todo parece indicar que el DDT-y los otros insecticidas ya no les hacen efecto a los piojos."

¿Cómo explicas que los insecticidas hace años les hicieran efecto a los piojos y ahora no? 
3. «RATONES"

A principios de siglo un naturalista realizó un experimento consistente en cortar, durante varias generaciones, la cola a unos ratones y ver cómo aparecía la descendencia. plicalo.

a. ¿Qué crees que sucedería al cabo de 20 generaciones? ¿Nacerían con cola o sin cola? Ex-

b. ¿Influiría algo en el resultado el que se entrenase a una lechuza para que cazase a los ratones por la cola, de forma que los que carecían de cola no fuesen atrapados? Explícalo.

\section{APENDICE 2}

En los extractos de respuestas los y las estudiantes del primer grupo figuran como $37,73 \ldots$ $y$ los del segundo como $37^{*}, 73^{*} \ldots$

- 19 «Ratones»: (a) «Tendrán cola, porque un ratón no es un ser tan infimo como una bacteria, y la mutación tendrá lugar en un proceso evolutivo a largo plazo, al cabo de mucho tiempo.»

- 27 «Bacterias»: "La estreptomicina en el medio activa algún gen y produce un cambio que afecta al genoma"

«Ratones»: (a y b) «Cortar la cola no afecta a los genes.»

-57* «Bacterias»: "Con la estreptomicina tendrán lugar cambios en el organismo, que al cabo de varias generaciones, se expresan en los genes.»

«Piojos»: «Los piojos se van acostumbrando poco a poco a las condiciones ambientales (...) durante algunas generaciones se adaptan a las condiciones en las que se desarrollan, en un ambiente con insecticida, de forma que finalmente se vuelven resistentes a él.»B 2 «Ratones»: (a y b) «Con cola. Es un cambio producido en la morfología del ratón, y no afecta a los genes en lo más minimo. No tendria la información para nacer sin cola." A

-61* «Bacterias»: «Las bacterias mutantes resistirán, mientras que las demás morirán.» A "Piojos»: "Si le echamos insecticida a un piojo, se sensibilizará y puede morir, pero si seguimos aplicándolo llegará un momento en que se volverá inmune." . B 3 "Ratones»: (a y b) "Con cola (...) porque la información genética es inalterable, incluso cuando el fenotipo se modifica."

\section{Extended Summary}

This paper is part of a more comprehensive study dealing with conceptual frameworks concerning the understanding of Evolution through Natural Selection, it explores the ideas obtained by University Students of Biology on the following issues:

- Persistence of alternative frameworks among this selected (by professional choice) group of individuals.

- Consistency in the use of the same frameworks in different tasks.

- Gender-related differences in the use of accepted and alternative frameworks.

Our initial hypotheses on each issue were:

- Existence of a large percentage of students with an alternative framework.

- A certain degree of consistence, but not in all individuals.

- A lesser use of the scientific accepted ideas by girls.

The students $(N=157)$ were provided with a set of three sheets of paper, each containing an application problem related to the subject. The words «evolution», «natural selection» or «adaptation» did 
not appear in the wording of any of these problems on different contexts, all being quite similar in construction.

For data analysis, answers to each task were grouped into mutually exclusive categories and subcategories. Three main categories were established:

A Explanations according to accepted scientific principles, or incorporating parts of it.

B Explanations based on post hoc adaptation of individuals and inheritance of acquired characteristics.

$C$ Other explanations including teleological, anthropomorphic, uncodeable and «does not answer».

The proportion of students using the accepted framework ranged from $59.23 \%$ in the «Mice» question to 31-18 in the «Lice» one. We feel that this low proportion is a matter for great concern. Moreover, a significant number of students gave teleological explanations, and some even offered anthropomorphic ones.

We expected a higher amount of students to use the accepted framework in the «Mice» task, since the organism is a vertebrate and the situation can be related to that of a human losing a limb. Some students established a contrast between morphological and physiological views in relation to genetic changes. Instead, we did not expect the contrast between responses to «Bacteria» and «Lice»; perhaps the difference in the use of framework relates to the «scientific» context as opposed to the "everyday» context of each problem, or else to the epistemological commitments that students hold -or do not.

Contingency tables were drawn for each pair of tasks. There is a positive correlation between the answers that use accepted and alternative frameworks in each pair, which challenges the idea of frameworks being ad hoc constructions.

In order to check the differences in the number of girls and boys using accepted frameworks, Chi-square proof were tabulated for each task. In all of them, boys used the accepted framework in a higher proportion than girls.

Considering that this is a selected group of individuals studying for a Biology degree, we think these results show the inefficiency of usual teaching strategies, and we suggest that new strategies are needed that take into account the alternative frameworks used by a large range of students. 